\title{
Toracotomía en el departamento de urgencias en una paciente con actividad eléctrica sin pulso no traumática: potencial ampliación del horizonte terapéutico de una intervención de alta especialidad
}

\author{
Emergency department thoracotomy in a patient with non-traumatic pulseless electrical \\ activity: potential widening of the therapeutic horizon of a high-specialty intervention
}

\section{Luis M. García-Núñez ${ }^{*}$, Irving I. Morales-Pogoda, Edgar F. Hernández-García',} Juan A. Rodríguez-Inurrigarro², Raúl García-Ramírez ${ }^{1}$ y Fernando F. Arcaute-Velázquez ${ }^{1}$

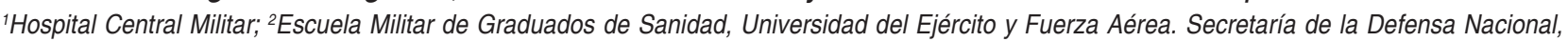
Ciudad de México, México

\begin{abstract}
Resumen
Instituida bajo recomendaciones objetivas, la toracotomía en el departamento de urgencias (TDU) se ha descrito como una maniobra quirúrgica salvatoria de la vida en pacientes traumatizados in extremis. Sin embargo, hay pocos reportes acerca de la experiencia con su empleo en la actividad eléctrica sin pulso no traumática. Describimos el caso de una paciente obstétrica exanguinada por sangrado masivo transoperatorio, en la que se realizó una TDU con un resultado óptimo para la vida y la función neurológica. Adicionalmente, evaluamos la literatura correspondiente al tema, que en lo mejor de nuestro conocimiento es crítica para expandir los protocolos de abordaje del ritmo de paro cardiaco no traumático en los hospitales de alto volumen.
\end{abstract}

PALABRAS CLAVE: Actividad eléctrica sin pulso no traumática. Toracotomía. Departamento de urgencias.

\begin{abstract}
Instituted under objective recommendations, Emergency Department Thoracotomy (EDT) has been described as a life-saving surgical maneuver in trauma patients arriving "in extremis." Nevertheless, there are few reports related to the experience regarding the use of the procedure in non-traumatic cardiopulmonary arrest. We describe the case of an obstetric patient exsanguinated by operative massive bleeding, where EDT was instituted reaching an optimal result for the survival and neurologic function. Additionally, we evaluate the literature related to this issue, which to the best of our knowledge, is critical to expand protocols of approach in non-traumatic cardiac arrest rhythm in high-volume hospitals.
\end{abstract}

KEY WORDS: Non-traumatic pulseless electrical activity. Thoracotomy. Emergency department.

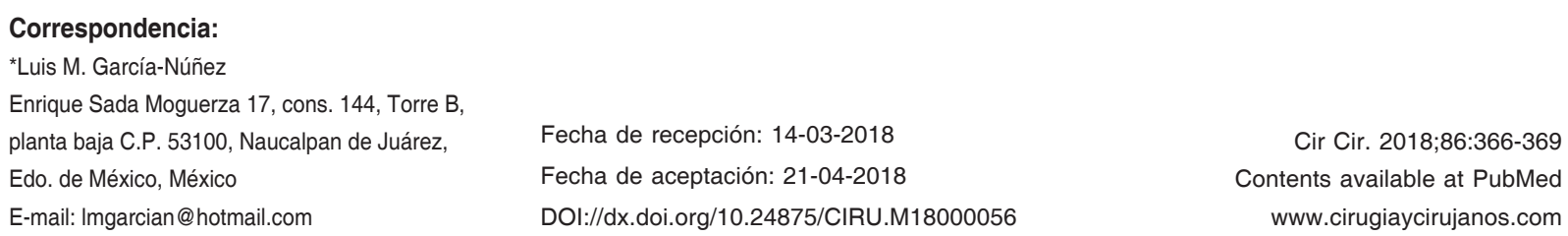




\section{Introducción}

La toracotomía en el departamento de urgencias (TDU) es un procedimiento heroico, que representa la última esperanza de vida para muchos pacientes que arriban críticamente lesionados e in extremis a los centros de trauma. Empleada bajo recomendaciones objetivas por un cirujano conocedor de la técnica, ha demostrado su gran utilidad en este escenario ${ }^{1-3}$.

Por mala fortuna, desde la popularización de las compresiones torácicas cerradas en la década de 1960, el masaje cardiaco directo con el tórax abierto cayó en desuso, haciendo que las compresiones cardiacas directas se practicaran rara vez fuera de los escenarios quirúrgicos ${ }^{4-6}$. Sin embargo, en los últimos 20 años se ha ganado gran experiencia con la reanimación con masaje cardiaco abierto, fundamentalmente debido al análisis de los resultados obtenidos con la práctica de la TDU en trauma.

Por lo anterior, la audacia quirúrgica, sustentada en un sólido conocimiento científico de la anatomofisiopatología del colapso cardiovascular y de la respuesta al procedimiento, ha fomentado el uso de la TDU en la actividad eléctrica sin pulso no traumática (AESPNT) ${ }^{4,6,7}$. Reportamos el caso de una paciente con AESPNT (por hemorragia obstétrica masiva) que fue sometida a TDU con un buen resultado para la vida y para la recuperación neurológica, lo cual abre presumiblemente un panorama alentador en el manejo de este tipo de casos.

\section{Descripción del caso clínico}

Mujer de 27 años, previamente sana, con antecedente de tres cesáreas y un parto vaginal (todos finalizados con carácter pretérmino) y embarazo no controlado de 26 semanas de gestación, quien arribó a una institución ginecoobstétrica por sangrado transvaginal de 9 horas de evolución. A su admisión se encontró en estado de choque (presión arterial $70 / 50 \mathrm{mmHg}$, frecuencia cardiaca 123 latidos/min; $\mathrm{SpO}_{2} 63 \%$ ), con alteración del sensorio, diaforética y con tez marmórea. En la exploración ginecológica se advirtió la vulva y la vagina con abundante sangrado, por lo cual se obvió el tacto vaginal, mientras que la frecuencia cardiaca fetal se ubicaba en 138 latidos/ min. En los estudios de laboratorio eran evidentes anemia profunda, plaquetopenia, acidosis metabólica y coagulopatía. Se practicó un ultrasonido transabdominal que demostró la presencia de placenta previa total. Del área de urgencias se trasladó al quirófano para terminar el embarazo por vía cesárea, por presentar placenta previa total sangrante.

En la sala de operaciones, con la citada intervención se obtuvo un producto masculino de 800 gramos, que se trasladó intubado a la unidad de cuidados intensivos neonatales. No fue posible retirar totalmente la placenta por acretismo, teniendo como consecuencia el sangrado transoperatorio continuo, lo cual activó los protocolos institucionales de transfusión masiva. La paciente presentó paro cardiaco, teniendo que realizar reanimación cardiopulmonar, a la que respondió después de 4 minutos. Debido a su condición crítica, se practicó una histerectomía obstétrica supracervical, resultando lesionada la vejiga y reparándola de forma primaria. Se empaquetó y la piel se cerró de forma temporal, para trasladarla por vía terrestre a la institución de referencia nacional (Hospital Central Militar), que es un hospital cercano de tercer nivel, con recursos humanos y materiales en plenitud. La pérdida estimada transoperatoria de sangre fue de $6300 \mathrm{ml}$ y el reemplazo volumétrico transoperatorio total se calculó en $13,600 \mathrm{ml}(2000 \mathrm{ml}$ de cristaloides, 17 concentrados eritrocitarios, 5 unidades de plasma fresco congelado y 3 pools plaquetarios).

Inmediatamente a su arribo al centro de referencia presentó un nuevo evento de paro cardiaco, documentando actividad eléctrica sin pulso por vía electrocardiográfica. Por ello, se realizó una TDU haciendo uso de una incisión de Spangaro (anterolateral izquierda). El cirujano operador encontró el corazón conteniendo sangre en las cuatro cámaras, realizó una pericardiotomía longitudinal e inició masaje cardiaco abierto, obteniendo una fibrilación ventricular como respuesta. De forma simultánea, se disecó no circunferencialmente y se realizó el pinzamiento de la aorta torácica descendente con unas pinzas de Crafoord-DeBakey, para redistribuir el volumen circulante efectivo hacia la circulación coronario-cerebral y limitar el sangrado subdiafragmático. Sin retraso, se aplicaron también los protocolos farmacológicos y de electroterapia para el tratamiento del ritmo fundamental, transformando esta fibrilación hacia una bradicardia sinusal (40 latidos/min). Se aplicó una dosis intraventricular directa de atropina $(1 \mathrm{mg})$ y de adrenalina (1 mg), llegando a un ritmo sinusal (72 latidos/min). Tras esto, se llevó al quirófano para reempaquetamiento abdominal, cierre temporal de la toracotomía con colocación de un tubo pleural izquierdo y manejo con abdomen abierto. El tiempo total de pinzamiento de la aorta fue menor de 15 minutos. 
En las primeras 10 horas de su estancia en la unidad de cuidados intensivos, la paciente presentó dos eventos de taquicardia ventricular con repercusión hemodinámica, que se manejaron exitosamente con los protocolos de soporte cardiovascular avanzado. Se instituyeron actividades intensivas de manejo de la coagulopatía multifactorial y terapia transfusional. Evolucionó hacia la mejoría y a las 36 horas de la TDU fue llevada al quirófano para el cierre definitivo de cavidades. Fue posible retirar la ventilación mecánica a las 20 horas de su readmisión a la unidad. Egresó de la unidad de cuidados críticos al sexto día de su admisión y el tubo pleural se retiró poco antes de su transferencia a la sala de hospitalización regular. La paciente fue egresada del hospital al día 12 de su arribo, en buen estado físico y con capacidad de autocuidado.

\section{Discusión}

Sabemos que la desfibrilación temprana y las compresiones cardiacas de alta calidad son posiblemente los aspectos más importantes que conducen hacia el retorno de la circulación espontánea en la AESPNT ${ }^{1,4,6}$. Sin embargo, en presencia de un ritmo de paro cardiaco traumático, cuando la pérdida de sangre desempeña un papel esencial en la fisiopatología del colapso, el control eficiente de la hemorragia se vuelve una faceta crítica en la terapéutica, que no debe ser perdida de vista por el cirujano operador ${ }^{7,8}$.

Bajo estos principios del soporte cardiovascular avanzado es posible que ciertos aspectos referentes a la práctica de la TDU en trauma sean aplicables a los pacientes con AESPNT. García-Núñez, et al. ${ }^{9}$, en un análisis crítico de la literatura referente a la cesárea perimortem, señalan que bajo condiciones óptimas las compresiones torácicas externas generan solo una tercera parte del gasto cardiaco calculado, mientras que la práctica del masaje cardiaco abierto promueve una fracción de expulsión del $60-80 \%$, con el subsecuente aumento del gasto cardiaco. Por otro lado, Kornhall y Dolven ${ }^{4}$, en un estudio similar relativo a la TDU en AESPNT, señalaron que las compresiones cardiacas abiertas resultan en un índice cardiaco medio de $13.1 \mathrm{l} / \mathrm{min} / \mathrm{m}^{2}$, comparado con $0.61 \mathrm{l} / \mathrm{min} / \mathrm{m}^{2}$ al practicar masaje cardiaco cerrado. En adición, las presiones medias de perfusión coronaria obtenidas con uno y otro método de reanimación son de $32.6 \pm 17.8$ y $7.3 \pm 5.7 \mathrm{mmHg}$, respectivamente.
Además, por el mismo motivo por el que es importante señalar al pinzamiento aórtico como un objetivo incuestionable de la TDU en trauma, al ser una maniobra invaluable para cohibir el sangrado infradiafragmático, es esencial apuntar al hecho de que en pacientes con AESPNT esta estrategia manifiesta uno de sus efectos benéficos más favorables a la sobrevida y la recuperación neurológica: la redistribución del volumen circulante efectivo residual hacia la circulación coronaria y cerebral. De esta forma, aunque la tasa de supervivencia de los pacientes traumatizados sujetos a TDU en el mejor de los casos se ha estimado históricamente en un $38 \%$, a partir de la inclusión protocolaria y oportuna del pinzamiento de la aorta torácica descendente se ha observado que en el $90 \%$ de los supervivientes no hay secuelas neurológicas adjudicadas al procedimiento. Sin embargo, hay que enfatizar que, debido a sus efectos perjudiciales, el pinzamiento aórtico no debe exceder una duración de 15 minutos, pues lapsos mayores se relacionan con una tasa de mortalidad que sobrepasa el $80-90 \% \%^{1-3,7,8,10,11}$.

Por lo que concierne a la terapia eléctrica, estudios procedentes de la atención del paro cardiopulmonar en pacientes críticamente lesionados, tanto en la arena urbana como en la militar, incluyendo algunos reanimados bajo la política de la TDU, no encuentran diferencia estadística entre las tasas de reversión de los ritmos fundamentales obtenidas con la desfibrilación interna o externa ${ }^{1-4,6-8,11,12}$, por lo cual, hasta este momento y con los elementos disponibles, no es posible determinar que la desfibrilación interna sea menos benéfica que su contraparte terapéutica externa.

En resumen, la TDU es un procedimiento que nació de la necesidad de atender a pacientes agónicos críticamente lesionados. Incluye dentro de su protocolo de institución aspectos esenciales que conciernen al masaje cardiaco, la terapia eléctrica y las maniobras de redistribución del flujo sanguíneo (pinzamiento de la aorta torácica descendente), que son aplicables plenamente al paciente con AESPNT y que pueden modificar significativamente el curso natural de la fisiopatología del colapso cardiovascular, promoviendo un beneficio en los índices pronósticos relativos a la sobrevida y la funcionalidad ${ }^{1-12}$. Sin embargo, la experiencia mundial con este procedimiento alentador es escasa y su verdadera utilidad solo podrá ser comprobada con el paso del tiempo y la ganancia de experiencia con su uso. 


\section{Conclusiones}

Aunque la TDU se instituye fundamentalmente en víctimas lesionadas en estado agónico, su empleo en AESPNT se está perfilando como una alternativa terapéutica posiblemente útil, lo cual puede valer su inclusión en los protocolos de atención cardiovascular avanzada. Sin embargo, la validación de esta apreciación debe aguardar a los resultados que sobre una gran casuística solo puede proporcionar a la comunidad científica un estudio multicéntrico con un apropiado diseño metodológico.

\section{Agradecimientos}

Los autores agradecen al personal médico y de enfermería adscrito a los Departamentos de Medicina Intensiva y Urgencias, así como al Jefe del Área de Cirugía del Hospital Central Militar, por proporcionar el apoyo asistencial y logístico requerido para la recuperación de la paciente. De la misma forma, se hace constar el papel que como colaboradores en sus áreas de especialidad tuvieron en la elaboración de este manuscrito los siguientes profesionales de la salud: Medicina del Enfermo en Estado Crítico, Mayor M.C. Deniria Alejandra Rugerio Trujillo y Mayor M.C. Miriam Seleste Ramírez Valdés; Urgencias
Médico-Quirúrgicas, Mayor M.C. Juan Antonio Ramos Martínez; y Cirugía de Trauma, Mayor M.C. Óscar Enrique Pérez Morales.

\section{Bibliografía}

1. García-Núñez LM, Núñez-Cantú $O$, Hernández-García EF, Petrone $P$, Asensio JA. Toracotomía en el departamento de urgencias: unificar conceptos para protocolizar su práctica. Rev Sanid Milit Méx. 2011;65:23-31.

2. García-Núñez LM. Toracotomía en el departamento de urgencias: errores graves... pero comunes. Ciruj Gen. 2011;33(Supl 1):S65-7.

3. García-Núñez LM, Hernández-García EF. Toracotomía en el departamento de urgencias: estado del arte para el cirujano general. PROACI. 2016;1(1):9-34. (Consultado el 3/11/2017.) Disponible en: http://secad. com.mx/wp-content/uploads/2016/08/PROACI-Articulo-muestra-Toracotomia-en-el-Departamento-de-Urgencias.pdf.

4. Kornhall DK, Dolven T. Resuscitative thoracotomies and open chest cardiac compressions in non-traumatic cardiac arrest. World $\mathrm{J}$ Emerg Surg. 2014;9:1-6.

5. Eldor J, Frankel DZN, Davidson JT. Open chest cardiac massage: a review. Resuscitation. 1988;16:155-62.

6. Alifimoff JK. Open versus closed chest cardiac massage in non-traumatic cardiac arrest. Resuscitation. 1987:15:13-21.

7. García-Núñez LM. Toracotomía en el departamento de urgencias. En: García-Núñez LM, editor. Cirugía avanzada en trauma (C.A.T.). Principios teóricos y de aplicación práctica. Ciudad de México: Graphimedic; 2016. p. 80-6.

8. Cothren CC, Moore EE. Emergency department thoracotomy for the critically injured patient: objectives, indications, and outcomes. World $\mathrm{J}$ Emerg Surg. 2006;1:1-13.

9. García-Núñez LM, Tëras J, Cabello-Pasini R, Loera-Torres MA. Cesárea perimortem. La visión del cirujano de trauma sustentada en una revisión multiinstitucional de la literatura. Rev Sanid Milit Méx. 2006;60:217-29.

10. Robertson C. Open-chest cardiac massage for non-traumatic cardiac arrest. Arch Emerg Med. 1987;4:207-10.

11. Robertson $\mathrm{C}$. The value of open chest $\mathrm{CPR}$ for non-traumatic cardiac arrest. Resuscitation. 1991;22:203-8.

12. Banghu A, Nepogodiev D, Bowley DM. Outcomes following military traumatic cardiorespiratory arrest: the role of surgery in resuscitation. Resuscitation. 2013;84:23-4. 